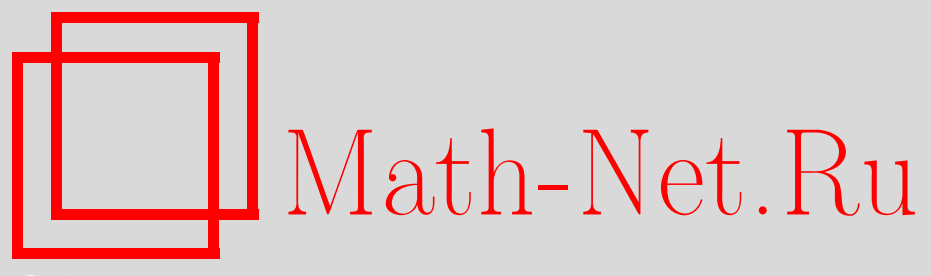

И. Р. Каюмов, Об одном неравенстве для универсального спектра интегральных средних, Матем. заметки, 2008, том 84, выпуск 1, 139-143

DOI: https://doi.org/10.4213/mzm4127

Использование Общероссийского математического портала Math-Net.Ru подразумевает, что вы прочитали и согласны с пользовательским соглашением http://www . mathnet.ru/rus/agreement

Параметры загрузки:

IP : 54.174 .149 .18

26 апреля 2023 г., 16:49:13

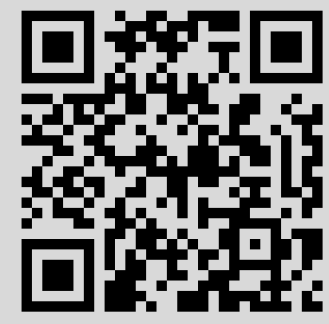




\section{Об одном неравенстве для универсального спектра интегральных средних}

\section{И. Р. Каюмов}

Пусть $S$ - класс функций, аналитических и однолистных в единичном круге, нормированных стандартным образом: $f(0)=0, f^{\prime}(0)=1$, и пусть

$$
B(t)=\sup \left\{\beta_{f}(t): f \in S\right\}
$$

- универсальный спектр интегральных средних, введенный Макаровым в [1]. Здесь

$$
\beta_{f}(t)=\limsup _{r \rightarrow 1} \frac{\ln \int_{0}^{2 \pi}\left|f^{\prime}\left(r e^{i \theta}\right)\right|^{t} d \theta}{|\ln (1-r)|}
$$

- спектр интегральных средних конформного отображения $f$. Универсальный спектр $B(t)$ отвечает за граничное поведение конформных отображений в круге, в частности, за метрические свойства гармонической меры на жордановых кривых, а также за искажение мер Хаусдорфа борелевских множеств на окружности при конформных отображениях круга (см., например, [2]-[4]).

Хорошо известно, что $B(t)$ является выпуклой функцией переменного $t$. Карлесоном и Макаровым показано [5], что

$$
B(t)=|t|-1
$$

при $t \leqslant t_{0}$ для некоторого $t_{0} \leqslant-2$ (далее будем считать, что $t_{0}$ является максимальным из возможных). Фенг и МакГрегор в работе [6] установили равенство

$$
B(t)=3 t-1 \quad \text { при } \quad t \geqslant \frac{2}{5} .
$$

Легко видеть, что из неравенства Гёльдера для интегралов следует, что

$$
\frac{B(p)}{|p|} \leqslant \frac{B(q)}{|q|} \quad \text { при } \quad|p| \leqslant|q|, \quad p q>0 .
$$

Основная цель данной работы - доказательство другого, в некотором смысле обратного, неравенства:

$$
\frac{B(q)}{|q|}-\frac{B(p)}{|p|} \leqslant \frac{1}{|p|}-\frac{1}{|q|} \quad \text { при } \quad|p| \leqslant|q|, \quad p q>0 .
$$

Доказательство основано на неравенстве, связывающем $L_{p^{-}}$и $L_{q}$-нормы полиномов, доказанном Зигмундом и Сеге в [7] (а по-существу, ранее, в более общем виде Никольским в [8]).

Введем подклассы класса $S$ :

$$
\begin{aligned}
S(n, 1) & =\left\{f \in S: f^{\prime}-\text { полином степени } n\right\}, \\
S(n,-1) & =\left\{f \in S: 1 / f^{\prime} \text { - полином степени } n\right\} .
\end{aligned}
$$

Определим новую величину, связанную с граничным поведением конформных отображений:

$$
\Gamma(t)=\limsup _{n \rightarrow \infty} \gamma_{n}(t),
$$

Работа выполнена при частичной поддержке Российского фонда фундаментальных исследований (грант № 08-01-00381).

(C) И. Р. КАюмов, 2008 
где

$$
\gamma_{n}(t)=\sup \left\{\frac{1}{\ln n} \ln \int_{0}^{2 \pi}\left|f^{\prime}\left(e^{i \theta}\right)\right|^{t} d \theta: f \in S(n, 1)\right\}
$$

в случае, когда $t \geqslant 0$,

$$
\gamma_{n}(t)=\sup \left\{\frac{1}{\ln n} \ln \int_{0}^{2 \pi}\left|f^{\prime}\left(e^{i \theta}\right)\right|^{t} d \theta: f \in S(n,-1)\right\}
$$

в случае отрицательного $t$. Справедлива

Теорема 1. Имеем $\Gamma(t)=B(t)$.

ДоказАтельство. Разберем подробно случай $t>0$. Сначала покажем, что $B(t) \leqslant$ $\Gamma(t)$. Для этого зафиксируем малое число $\varepsilon>0$. Из определения универсального спектра следует, что найдется однолистная функция $f \in S$ такая, что

$$
\beta_{f}(t)>B(t)-\varepsilon
$$

Нетрудно показать, что найдется последовательность натуральных чисел $n_{k}$ такая, что

$$
\beta_{f}(t)=\lim _{k \rightarrow \infty} \frac{\ln \int_{0}^{2 \pi}\left|f^{\prime}\left(r_{k} e^{i \theta}\right)\right|^{t} d \theta}{\ln \left(1 /\left(1-r_{k}\right)\right)},
$$

где $r_{k}=1-5 \ln n_{k} / n_{k}$. Пусть $a_{j}$ - тейлоровские коэффициенты $f(z)$. В силу хорошо известной теоремы Де Бранжа [9] имеет место оценка $\left|a_{j}\right| \leqslant j$. Покажем, что полином $p(z)=\sum_{j=1}^{n_{k}+1} a_{j} r_{k}^{j} z^{j}$ однолистен в круге $|z|<1$ начиная, с некоторого $k$. Для этого возьмем произвольные точки $z_{1}$ и $z_{2}$ на окружности $|z|=1$. Далее имеем

$$
\begin{aligned}
\left|p\left(z_{1}\right)-p\left(z_{2}\right)\right| & >\left|f\left(r_{k} z_{1}\right)-f\left(r_{k} z_{2}\right)\right|-\left|\sum_{j=n_{k}+2}^{\infty} a_{j} r_{k}^{j}\left(z_{1}^{j}-z_{2}^{j}\right)\right| \\
& \geqslant \frac{1-r_{k}}{1+r_{k}}\left|z_{1}-z_{2}\right|-\left|z_{1}-z_{2}\right|\left|\sum_{j=n_{k}+2}^{\infty} j^{2} r_{k}^{j}\right| .
\end{aligned}
$$

В последнем неравенстве мы использовали одну из теорем искажения [10]:

$$
\frac{\left|f\left(r z_{1}\right)-f\left(r z_{2}\right)\right|}{\left|z_{1}-z_{2}\right|} \geqslant \frac{(1-r)}{(1+r)}
$$

а также упоминавшееся выше неравенство де Бранжа $\left|a_{j}\right| \leqslant j$.

Для доказательства однолистности полинома $p$ осталось показать, что

$$
\frac{1-r_{k}}{2}-\sum_{j=n_{k}+2}^{\infty} j^{2} r_{k}^{j}>0
$$

начиная с некоторого $k$. Имеем

$$
\begin{aligned}
\frac{1-r_{k}}{2}-\sum_{j=n_{k}+2}^{\infty} j^{2} r_{k}^{j} & =5 \frac{\ln n_{k}}{n_{k}}-\sum_{j=n_{k}+2}^{\infty} j^{2}\left(1-5 \frac{\ln n_{k}}{n_{k}}\right)^{j} \\
& \geqslant 5 \frac{\ln n_{k}}{n_{k}}-\sum_{j=n_{k}+2}^{\infty} j^{2} n_{k}^{-5 j / n_{k}}
\end{aligned}
$$


Последнее выражение будет, очевидно, строго положительно, начиная с некоторого $k$. Итак, $p(z) / r_{k} \in S\left(n_{k}, 1\right)$. Отсюда следует, что

$$
\begin{aligned}
\gamma_{n_{k}}(t) & \geqslant \frac{1}{\ln n_{k}} \ln \int_{0}^{2 \pi}\left|p^{\prime}\left(e^{i \theta}\right)\right|^{t} d \theta \geqslant \frac{1}{\ln n_{k}} \ln \int_{0}^{2 \pi}\left|p^{\prime}\left(r_{k} e^{i \theta}\right)\right|^{t} d \theta \\
& =\frac{1}{\ln n_{k}} \ln \int_{0}^{2 \pi}\left|f^{\prime}\left(r_{k} e^{i \theta}\right)-\sum_{j=n_{k}+2}^{\infty} j a_{j} r_{k}^{j-1} e^{i(j-1) \theta}\right|^{t} d \theta .
\end{aligned}
$$

В силу положительности (3)

$$
\left|\sum_{j=n_{k}+2}^{\infty} j a_{j} r_{k}^{j-1} e^{(j-1) \theta}\right| \leqslant 5 \frac{\ln n_{k}}{n_{k}} .
$$

Поэтому

$$
\Gamma(t)=\limsup _{n \rightarrow \infty} \gamma_{n}(t) \geqslant \limsup _{k \rightarrow \infty} \frac{1}{\ln n_{k}} \ln \int_{0}^{2 \pi}\left|f^{\prime}\left(r_{k} e^{i \theta}\right)\right|^{t} d \theta=\beta_{f}(t) .
$$

Теперь из $(2)$ следует, что $\Gamma(t) \geqslant B(t)-\varepsilon$. Устремляя $\varepsilon$ к нулю, заключаем, что $\Gamma(t) \geqslant$ $B(t)$. Эти же рассуждения с небольшими изменениями показывают, что $\Gamma(t) \geqslant B(t)$ и при отрицательных значениях $t$.

Покажем теперь, что $\Gamma(t) \leqslant B(t)$. Для этого нам понадобится один результат из работы Карлесона и Джонса [3], который утверждает, что

$$
B(t)=\limsup _{r \rightarrow 1} \frac{\ln \sup _{f \in S} \int_{0}^{2 \pi}\left|f^{\prime}\left(r e^{i \theta}\right)\right|^{t} d \theta}{|\ln (1-r)|} .
$$

Пусть полином $p_{n}(z)$ максимизирует величину

$$
\frac{1}{\ln n} \ln \int_{0}^{2 \pi}\left|f^{\prime}\left(e^{i \theta}\right)\right|^{t} d \theta
$$

в классе $S(n, 1)$, т.е.

$$
\gamma_{n}(t)=\frac{1}{\ln n} \ln \int_{0}^{2 \pi}\left|p_{n}^{\prime}\left(e^{i \theta}\right)\right|^{t} d \theta
$$

Существование такого полинома гарантируется компактностью класса $S$. Из равенства (4) следует, что

$$
B(t) \geqslant \limsup _{n \rightarrow \infty} \frac{\ln \int_{0}^{2 \pi}\left|p_{n}^{\prime}\left((1-1 / n) e^{i \theta}\right)\right|^{t} d \theta}{\ln n} .
$$

Из однолистности полинома $p_{n}$ следует, что $p_{n}^{\prime}(z) \neq 0$ в круге $|z|<1$, что влечет существование чисел $z_{1}, z_{2}, \ldots, z_{n}$ таких, что $\left|z_{j}\right| \leqslant 1$ для всех $j$ и

$$
p_{n}^{\prime}(z)=\prod_{j=1}^{n}\left(1+z_{j} z\right)
$$

Из легко проверяемого неравенства

$$
\left|\frac{1+z}{1+r z}\right| \leqslant \frac{2}{1+r}, \quad|z| \leqslant 1, \quad 0 \leqslant r \leqslant 1,
$$

следует, что

$$
\begin{aligned}
\left|p_{n}^{\prime}\left(e^{i \theta}\right)\right| & \leqslant\left|p_{n}^{\prime}\left(\left(1-\frac{1}{n}\right) e^{i \theta}\right)\right| \prod_{j=1}^{n} \frac{2}{2-1 / n} \\
& =\frac{1}{(1-1 /(2 n))^{n}}\left|p_{n}^{\prime}\left(\left(1-\frac{1}{n}\right) e^{i \theta}\right)\right| \leqslant 2\left|p^{\prime}\left(\left(1-\frac{1}{n}\right) e^{i \theta}\right)\right| .
\end{aligned}
$$


Подставляя последнее неравенство в (5), получаем

$$
\begin{aligned}
B(t) & \geqslant \limsup _{n \rightarrow \infty} \frac{\ln 2^{-t} \int_{0}^{2 \pi}\left|p_{n}^{\prime}\left(e^{i \theta}\right)\right|^{t} d \theta}{\ln n}=\limsup _{n \rightarrow \infty} \frac{\ln \int_{0}^{2 \pi}\left|p_{n}^{\prime}\left(e^{i \theta}\right)\right|^{t} d \theta}{\ln n} \\
& =\limsup _{n \rightarrow \infty} \gamma_{n}(t)=\Gamma(t) .
\end{aligned}
$$

Схема доказательства в случае отрицательных $t$ почти идентична случаю $t>0$ и мы на ней не останавливаемся.

Теорема 1 доказана.

Теорема 2. Предположим, что $р$ и $q$-вещественные числа одного знака $u|p| \leqslant|q|$. Тогда имеет место неравенство

$$
\frac{B(q)}{|q|}-\frac{B(p)}{|p|} \leqslant \frac{1}{|p|}-\frac{1}{|q|} .
$$

ДокАЗАТЕЛЬСтво. Мы снова разберем подробно лишь случай $t>0$. Для произвольных полиномов $g$ степени $n$ в (5) показано, что существуют числа $C_{p, q}$, зависящие только от $p$ и $q$ такие, что при $0<p<q$ имеет место неравенство

$$
\left(\int_{0}^{2 \pi}\left|g\left(e^{i \theta}\right)\right|^{q} d \theta\right)^{1 / q} \leqslant C_{p, q} n^{1 / p-1 / q}\left(\int_{0}^{2 \pi}\left|g\left(e^{i \theta}\right)\right|^{p} d \theta\right)^{1 / p} .
$$

По определению

$$
\Gamma(q)=\limsup _{n \rightarrow \infty} \sup _{f \in S(n, 1)} \frac{1}{\ln n} \ln \int_{0}^{2 \pi}\left|f^{\prime}\left(e^{i \theta}\right)\right|^{q} d \theta .
$$

Поскольку $f^{\prime}$ - полином степени $n$, в силу (7)

$$
\Gamma(q) \leqslant \limsup _{n \rightarrow \infty} \sup _{f \in S(n, 1)} \frac{1}{\ln n} \ln \left\{n^{q / p-1} C_{p, q}\left(\int_{0}^{2 \pi}\left|f^{\prime}\left(e^{i \theta}\right)\right|^{p} d \theta\right)^{q / p}\right\}=\frac{q}{p}-1+\frac{q}{p} \Gamma(p),
$$

откуда

$$
\frac{\Gamma(q)}{q}-\frac{\Gamma(p)}{p} \leqslant \frac{1}{p}-\frac{1}{q},
$$

что в силу теоремы 1 доказывает теорему 2 в случае положительных $p$ и $q$. Оставшийся случай доказывается аналогично.

Теорема 2 доказана.

Отметим, что в случае когда $p$ и $q$ не превосходят $t_{0}$ или лежат в интервале $[2 / 5,+\infty)$, вышеупомянутые результаты Карлесона, Макарова, Фенга и МакГрегора показывают, что неравенство в (6) заменяется равенством.

Приведем одно следствие неравенства (1). Макаровым ([4], [11]) доказано, что для любой функции $f \in S$, любого измеримого по Борелю множества $A \subset \partial \mathbb{D}$ и любого $s>0$ справедливо неравенство

$$
\operatorname{dim} f(A) \geqslant \frac{s \operatorname{dim} A}{B(-s)+s+1-\operatorname{dim} A},
$$

где $\operatorname{dim} A$ - хаусдорфова размерность множества $A$.

Если $\operatorname{dim} A=1$, то в неравенстве (8) выбор $s=0$ максимизирует правую часть этого неравенства и дает неулучшаемую оценку $\operatorname{dim} f(A) \geqslant 1$, доказанную Макаровым [4]. При $\operatorname{dim} A<1$ параметр $s$ нужно выбирать строго положительным, поскольку $B(s)=$ $c s^{2}+O\left(s^{3}\right)$ при малых $t$ (см. [11]). 
Предположим теперь, что хаусдорфова размерность $A$ достаточно мала. Тогда из неравенства (8) легко следует неравенство

$$
\operatorname{dim} f(A) \geqslant \frac{\operatorname{dim} A}{(B(-s)+1) / s+1}+\frac{\operatorname{dim}^{2} A}{s((B(-s)+1) / s+1)^{2}}+O\left(\operatorname{dim}^{3} A\right) .
$$

Полагая $s=\left|t_{0}\right|$ и пользуясь асимптотическим неравенством (9), Карлесон и Макаров [5] доказали неравенство

$$
\operatorname{dim} f(A) \geqslant \frac{\operatorname{dim} A}{2}+\frac{\operatorname{dim}^{2} A}{4\left|t_{0}\right|}+O\left(\operatorname{dim}^{3} A\right) .
$$

Возникает естественный вопрос: можно ли улучшить оценку (10) выбирая другой параметр $s$ в неравенстве (9)?

Оказывается, что неравенство (10) является оптимальным с точки зрения неравенств (8) и (9). Справедливо

ПреДЛоЖениЕ. Для достаточно мальх $\operatorname{dim}$ A имеет место соотношение

$$
\max _{s>0} \frac{s \operatorname{dim} A}{B(-s)+s+1-\operatorname{dim} A}=\frac{\operatorname{dim} A}{2}+\frac{\operatorname{dim}^{2} A}{4\left|t_{0}\right|}+O\left(\operatorname{dim}^{3} A\right) .
$$

ДокАзАТЕльство. Ясно, что параметр $s$ в (9) нужно выбрать таким образом, чтобы функция $(B(-x)+1) / x$ была минимальна в точке $s$. Из теоремы 2 следует, что функция $(B(-x)+1) / x$ не возрастает при $x>0$. Следовательно, нужно выбрать $s \geqslant\left|t_{0}\right|$. В этом случае $B(-s)=|s|-1$ и несложный анализ неравенства (9) показывает, что оптимальным является параметр $s=\left|t_{0}\right|$.

\section{СПИСОК ЦИТИРОВАННОЙ ЛИТЕРАТУРЫ}

[1] Н. Г. Макаров, Алгебра и анализ, 10:2 (1998), 1-62. [2] H. Hedenmalm, I. Kayumov, Proc. Amer. Math. Soc., 135:7 (2007), 2235-2248. [3] L. Carleson, P. W. Jones, Duke Math. J., 66:2 (1992), 169-206. [4] N. G. Makarov, Ark. Mat., 25:1 (1987), 41-89. [5] L. Carleson, N. G. Makarov, Ark. Mat., 32:1 (1994), 33-62. [6] J. Feng, T. H. MacGregor, J. Anal. Math., 29:1 (1976), 203-231. [7] G. Szegö, A. Zygmund, J. Anal. Math., 3:1 (1954), 225-244. [8] С. М. Никольский, Докл. АН СССР, 76 (1951), 785-788. [9] L. A. de Branges, Acta Math., 154:1-2 (1985), 137-152. [10] Дж. Дженкинс, Однолистные функции и конформные отображения, ИЛ, М., 1962. [11] Ch. Pommerenke, Boundary Behaviour of Conformal Maps, Grundlehren Math. Wiss., 299, Springer-Verlag, Berlin, 1992.

\section{И. Р. Каюмов}

Поступило

НИИ математики и механики им. Н. Г. Чеботарева

10.10 .2007

Казанского государственного университета 